\title{
Effect of Rotation on Thermosolutal Convection in Visco-elastic Nanofluid with Porous Medium
}

\author{
D.O.I - 10.51201/Jusst12650 \\ http://doi.org/10.51201/Jusst12650 \\ Sudhir Kumar Pundir, Mukul Kumar*, Rimple Pundir \\ Department of Mathematics \\ S. D. (P.G.) College Muzaffarnagar, U.P.- 251001, India
}

\begin{abstract}
In this paper, we studied the rotation effect on the thermosolutal convection in visco-elastic nanofluid in the presence of porous medium using Walters` (model B'). To solve the conservation equation, we used the normal mode technique and Galerkin weighted residual method. For stationary convection, the onset criterion derived analytically and experiential that visco-elastic nanofluid behaves as a regular Newtonian nanofluid. The effect of rotation, thermo-nanofluid Lewis number, thermosolutal Lewis number and solutal Rayleigh number analyze analytically and graphically.
\end{abstract}

Keywords- Nanofluid; thermosolutal instability; Walters`(model B`); porous medium; rotation.

\section{Introduction}

The problem of thermosolutal convection in porous medium has motivated during the last few decades, because it has various applications in soil science, oceanography, engineering, astrophysics etc. The thermal instability for Newtonian fluid with hydrodynamic and hydromagnetic assumptions was discussed by Chandrasekhar [2]. Kuznetsov and Nield [6] investigated theoretically the expression for thermal Rayleigh number, the condition for oscillatory motions derived and the instability of nanofluids using conservation equation. The nanofluid was firstly used by Choi [4] in regular fluid with nanometer sized particles for the colloidal suspension. The nanoparticles size is less than $100 \mathrm{~nm}$ in a base fluid, in nanofluids, for instance water, engine oils, ethanol are commonly used as base fluids. The materials of nanoparticles may be in use as nitrides (AIN, SiN), metal carbides ( $\mathrm{SiC})$, oxide ceramics $\left(\mathrm{Al}_{2} \mathrm{O}_{3}, \mathrm{Cuo}\right)$ or metals $(\mathrm{Cu}, \mathrm{Al})$. Kuznetsov and Nield was studied to the convection in a binary nanofluid layer in porous medium. The thermosolutal and thermal instability problems for Walters` (model B') with elastico-viscous fluid in a porous medium studied by Rana and Sharma [9]. Gupta et al. studied the effect of horizontal magnetic field on nanofluid convection [5]. Pundir et al. studied on the onset of thermosolutal convection of an elastico-viscous nanofluid in porous medium in presence of magnetic field [8]. Sharma and Gupta studied double diffusive nanofluid convection in porous medium with rotation using Darcy-Brinkman model [10]. The effect of rotation on nanofluid convection in porous was studied by Chand and Rana [3]. We are investigate the effect of rotation on thermosolutal convection of visco-elastic nanofluid presence of porous medium using Walters` (model B`). The coriolis force term is added in the momentum equation due to the presence of rotation so we introduce a non-dimensional rotation parameter Taylor number. The problem is analized with normal mode technique and Galerkin weighted residual method. The effect of rotation, thermo-nanofluid Lewis number, thermosolutal Lewis number and solutal Rayleigh number analyze graphically. 


\section{Mathematical Model}

Here we regard a rotating horizontal layer with thickness $d$ and angular velocity $\boldsymbol{\Omega}$ of Walters` (model B') elastico-viscous nanofluid situated between the plates $z=0$ and $z=d$. The fluid layer is heated from lower layer and working upwards direction with a gravity force $\boldsymbol{g}=(0,0,-g)$. Temperature $T_{D}$, concentration $C_{D}$ and volumetric fraction $\varphi_{D}$ of nanoparticle, at the lower boundary and upper boundary are taken to be $T_{1}$ and $T_{0}, C_{1}$ and $C_{0}, \varphi_{1}$ and $\varphi_{0}$ respectively, with $T_{1}>T_{0}, C_{1}>C_{0}$ and $\varphi_{0}>\varphi_{1}$. The governing equation for Walters'(model B') elastico-viscous nanofluid in porous medium as given by Yadav et al. [11] and Nield and Kuznetsov [7] are:

$\nabla \boldsymbol{q}_{D}=0$

$\frac{\rho}{\varepsilon} \frac{\partial \boldsymbol{q}_{D}}{\partial t}=-\nabla p+\left(\varphi_{D} \rho_{p}+\left(1-\varphi_{D}\right)\left\{\rho\left(1-\alpha_{T}\left(T_{D}-T_{0}\right)-\alpha_{C}\left(C_{D}-C_{0}\right)\right)\right\}\right) \boldsymbol{g}-\frac{1}{\boldsymbol{k}}\left(\mu-\mu^{\prime} \frac{\partial}{\partial t}\right) \boldsymbol{q}_{D}+\mu \nabla^{2} \boldsymbol{q}_{D}+$ $\frac{2 \rho}{\varepsilon}\left(\boldsymbol{q}_{D} \times \Omega\right)$

where $\boldsymbol{q}_{D}, p, \mu, \mu^{\prime}, \boldsymbol{g}, k, \rho, \varepsilon, \varphi_{D}, \alpha_{C}$, and $\alpha_{T}$ denoted by the Darcy velocity, hydrostatic pressure, viscosity, viscoelasticity, acceleration attainable to gravity, medium permeability, density, porosity, volume fraction of nanoparticles, solute concentration and coefficient of thermal expansion respectively.

For the nanofluid, the equation of thermal energy is given as:

$\left(\rho_{c}\right)_{m} \frac{\partial T_{D}}{\partial t}+\rho_{c} \boldsymbol{q}_{D} \cdot \nabla T_{D}=k_{m} \nabla^{2} T_{D}+\varepsilon\left(\rho_{c}\right)_{p}\left[D_{B} \nabla \varphi_{D} \cdot \nabla T_{D}+\frac{D_{T}}{T_{0}} \nabla T_{D} \cdot \nabla T_{D}\right]+\rho_{c} D_{T C} \nabla^{2} C_{D}$

where $D_{T C}$ is a Dufour diffusivity, $k_{m}$ is thermal conductivity, $\left(\rho_{c}\right)_{p}$ is the heat capacity of nanoparticles and $\left(\rho_{c}\right)_{m}$ is heat capacity of the fluid in porous medium.

For the nanoparticles, the continuity equation given by Biongiorno [1] as:

$\frac{\partial \varphi_{D}}{\partial t}+\frac{\boldsymbol{q}_{D}}{\varepsilon} \cdot \nabla \varphi_{D}=D_{B} \nabla^{2} \varphi_{D}+\frac{D_{T}}{T_{0}} \nabla^{2} T_{D}$

where $D_{B}$ and $D_{T}$ are the Brownian diffusion coefficient and the thermoporetic diffusion coefficient, respectively.

The equation of conservation of solute concentration is given as:

$\frac{\partial C_{D}}{\partial t}+\frac{1}{\varepsilon} \boldsymbol{q}_{D} \cdot \nabla C_{D} \quad=D_{S} \nabla^{2} C_{D} \quad+D_{C T} \nabla^{2} T_{D}$

where $D_{C T}$ and $D_{S}$ are Soret type diffusivity and the solute diffusivity of porous medium.

The boundary conditions are given as:

$q=0, \quad T_{D}=T_{1}, \quad \varphi_{D}=\varphi_{1}, \quad C_{D}=C_{1}$ at $z=0$

$q=0, \quad T_{D}=T_{0}, \quad \varphi_{D}=\varphi_{0}, \quad C_{D}=C_{0}$ at $z=d$

We establish nondimensional variables as:

$$
\begin{gathered}
\left(x^{*}, y^{*}, z^{*}\right)=\frac{(x, y, z)}{d}, \quad \boldsymbol{q}^{*}=\boldsymbol{q}_{D} \frac{d}{\alpha_{m}}, \quad t^{*}=\frac{t \alpha_{m}}{\sigma d^{2}}, p^{*}=\frac{p k}{\mu \alpha_{m}}, \quad \phi^{*}=\frac{\varphi_{D}-\varphi_{1}}{\varphi_{0}-\varphi_{1}}, \\
T^{*}=\frac{T_{D}-T_{0}}{T_{1}-T_{0}}, C^{*}=\frac{C_{D}-C_{0}}{C_{1}-C_{0}},
\end{gathered}
$$

where $\alpha_{m}=\frac{k_{m}}{\rho_{c}}, \quad \sigma=\frac{\left(\rho_{c}\right)_{m}}{\rho_{c}}$.

Dropping the star $(*)$ for simplification. Equations (1) and equation (5) to (10) reduce in non-dimensional form:

$\nabla \boldsymbol{q}=0$

$0=-\nabla p-\left(1-F \frac{\partial}{\partial t}\right) q+P l \nabla^{2} q-R_{m} \hat{k}-R_{n} \varphi \hat{k}+R_{D} T \hat{k}+\frac{R s}{L s} C \hat{k}+\sqrt{T a}(q \times \hat{k})$ 
$\frac{\partial T}{\partial t}+\boldsymbol{q} \cdot \nabla T=\nabla^{2} T+\frac{N_{B}}{L n} \nabla \varphi \cdot \nabla T+\frac{N_{D} N_{B}}{L n} \nabla T \cdot \nabla T+S_{T C} \nabla^{2} C$

$\frac{1}{\sigma} \frac{\partial \varphi}{\partial t}+\frac{1}{\varepsilon} \boldsymbol{q} \cdot \nabla \varphi=\frac{1}{L n} \nabla^{2} \varphi+\frac{N_{D}}{L n} \nabla^{2} T$

$\frac{1}{\sigma} \frac{\partial C}{\partial t}+\frac{1}{\varepsilon} \boldsymbol{q} \cdot \nabla C=\frac{1}{L s} \nabla^{2} C+S_{C T} \nabla^{2} T$

where the dimensionless parameters are:

Thermosolutal Lewis number $L s=\frac{\alpha_{m}}{D_{S}}$, Thermonanofluid Lewis number $L n=\frac{\alpha_{m}}{D_{B}}$, Kinematic viscoelastic parameter $F=\frac{\mu^{\prime} \alpha_{m}}{\mu \sigma d^{2}}$, Density Rayleigh number $R_{m}=\frac{\rho_{p} \varphi_{1}+\rho\left(1-\varphi_{1}\right) g k d}{\mu \alpha_{m}}$, Nanoparticle Rayleigh number $R_{n}=$ $\frac{\left(\rho_{p}-\rho\right)\left(\varphi_{0}-\varphi_{1}\right) g k d}{\mu \alpha_{m}}, \quad$ Thermal Rayleigh Darcy number $R_{D}=\frac{\rho \alpha_{T}\left(T_{1}-T_{0}\right) g k d}{\mu \alpha_{m}}$, Solutal Rayleigh number $R_{S}=$ $\frac{\rho \alpha_{C}\left(C_{1}-C_{0}\right) g k d}{\mu D_{S}}$, Dimensionless medium permeability $P l=\frac{k}{d^{2}}, \quad$ Modified diffusivity ratio $N_{D}=\frac{D_{T}\left(T_{1}-T_{0}\right)}{D_{B} T_{0}\left(\varphi_{0}-\varphi_{1}\right)}$, Modified particle density increment $N_{B}=\frac{\left(\rho_{c}\right)_{p}\left(\varphi_{1}-\varphi_{01}\right)}{\rho_{c}}$, Soret parameter $S_{C T}=\frac{D_{C T}\left(T_{1}-T_{0}\right)}{\alpha_{m}\left(C_{1}-C_{0}\right)} \quad$ Dufour parameter $S_{T C}=\frac{D_{T C}\left(C_{1}-C_{0}\right)}{\alpha_{m}\left(T_{1}-T_{0}\right)}$, Taylor number $T a=\left(\frac{2 \Omega d^{2} \rho}{\varepsilon \mu}\right)^{2}$.

The dimensionless boundary conditions are:

$w=0, \quad T=1, \quad \varphi=1, \quad C=0$ at $z=0$

$w=0, \quad T=0, \quad \varphi=0, \quad C=1$ at $z=1$

\subsection{Basic states and its solutions}

The basic state of nanofluid is assumed and does not depend on time and describes as:

$$
\boldsymbol{q}(u, v, w)=0, p=p(z), \quad T=T_{i}(z), \varphi=\varphi_{i}(z), C=C_{i}(z)
$$

The basic variable represented by subscript $i$.

The equations (8) to (12) with boundary conditions (13) and (14) gives the solution:

$T_{i}=1-z, \quad C_{i}=1-z$ and $\varphi_{i}=z$.

\subsection{Perturbation solutions}

We introduced small perturbations on the basic state for the investigate the stability of the system and write $\boldsymbol{q}^{*}=0+\boldsymbol{q}^{\prime}(u, v, w), T^{*}=(1-z)+T^{\prime}, C^{*}=(1-z)+C^{\prime}, \varphi^{*}=z+\varphi^{\prime}, \quad p^{*}=p_{i}+p^{\prime}$

Using equation (16) in equations (8) to (12) and linearise by disuse the multiplication of the prime quantities, and after dipping the dash $(')$, we get the subsequent equations:

$\nabla \boldsymbol{q}=0$

$0=-\nabla p-\left(1-F \frac{\partial}{\partial t}\right) q+P l \nabla^{2} q-R_{n} \varphi \hat{k}+R_{D} T \hat{k}+\frac{R s}{L s} C \hat{k}+\sqrt{T a}(q \times \hat{k})$

$\frac{\partial T}{\partial t}-w=\nabla^{2} T+\frac{N_{B}}{L n}\left(\frac{\partial \mathrm{T}}{\partial \mathrm{z}}-\frac{\partial \varphi}{\partial \mathrm{z}}\right)-2 \frac{N_{D} N_{B}}{L n} \frac{\partial \mathrm{T}}{\partial \mathrm{z}}+S_{T C} \nabla^{2} C$

$\frac{1}{\sigma} \frac{\partial \varphi}{\partial t}+\frac{1}{\varepsilon} w=\frac{1}{L n} \nabla^{2} \varphi+\frac{N_{D}}{L n} \nabla^{2} T$

$\frac{1}{\sigma} \frac{\partial C}{\partial t}-\frac{1}{\varepsilon} w=\frac{1}{L s} \nabla^{2} C+S_{C T} \nabla^{2} T$

and boundary conditions are:

$w=0, \quad T=0, \quad \varphi=0, \quad C=0 \quad$ at $\quad z=0$ and $z=1$.

$R_{m}$ is not involved in these because $R_{m}$ is presently a estimate of basic static pressure gradient. So by operating equation (18) with $\hat{k}$.curl.curl, we get: 


$$
\left[-\left(1-F \frac{\partial}{\partial t}\right)+P l\right] \nabla^{2} w+R_{D} \nabla_{H}^{2} T-R_{n} \nabla_{H}^{2} \varphi+\frac{R s}{L s} \nabla_{H}^{2} C+T a \frac{\partial^{2} w}{\partial z^{2}}=0
$$

where $\nabla_{H}^{2}=\frac{\partial^{2}}{\partial x^{2}}+\frac{\partial^{2}}{\partial y^{2}} \quad$ and $\nabla^{2}=\frac{\partial^{2}}{\partial x^{2}}+\frac{\partial^{2}}{\partial y^{2}}+\frac{\partial^{2}}{\partial z^{2}}$

\section{Normal mode analysis}

The disturbances analyzing by normal mode analysis as follow:

$[w, T, C, \varphi]=[W(z), \Theta(z), \Gamma(z), \phi(z)] \exp \left(i k_{x} x+i k_{y} y+n t\right)$

where $n$ is the growth rate and $k_{x}$ and $k_{y}$ are the wave number along $x$ and $y$ directions, respectively.

Using equation (24) in equations(27) to (29) and equation (23), we get;

$\left[\{-(1-n F)+P l\}\left(D^{2}-a^{2}\right)+T a D^{2}\right] W-R_{D} a^{2} \Theta-\frac{R s}{L S} a^{2} \Gamma+a^{2} R_{n} \phi=0$

$W+\left[\left(D^{2}-a^{2}\right)-n+\varepsilon \frac{N_{B}}{L n} D-2 \varepsilon \frac{N_{D} N_{B}}{L n} D\right] \Theta+S_{T C}\left(D^{2}-a^{2}\right) \Gamma-\frac{N_{B}}{L n} D \phi=0$

$\frac{W}{\varepsilon}-\frac{N_{D}}{L n}\left(D^{2}-a^{2}\right) \Theta+\left[\frac{n}{\sigma}-\frac{D^{2}-a^{2}}{L n}\right] \phi=0$

$\frac{W}{\varepsilon}+S_{C T}\left(D^{2}-a^{2}\right) \Theta+\left(\frac{D^{2}-a^{2}}{L s}-\frac{n}{\sigma}\right) \Gamma=0$

where $D=\frac{d}{d z}$ and $a^{2}=k_{x}^{2}+k_{y}^{2}$ is the dimensionless ensuing wave number and the boundary conditions in view of normal mode are:

$W=D^{2} W=\Gamma=\Theta=\phi=0$ at $z=0$ and $z=1$

\section{Linear stability analysis}

The eigen functions $f_{i}(z)$ corresponding to the eigen values problem (35) to (38) are $f_{j}=\sin (\pi z)$. the corresponding solutions are:

$W=W_{0} \sin (\pi z), \quad \Theta=\Theta_{0} \sin (\pi z), \quad \Gamma=\Gamma_{0} \sin (\pi z), \phi=\phi_{0} \sin (\pi z)$

The linear system has a solutions if and only if

$R_{D}=\frac{1}{J^{2} \sigma \varepsilon+n \varepsilon L S-S_{T C} J^{2} L s \sigma}\left[\frac{\left.(-\{-(1-n F)+P l\}\}^{2}+\pi^{2} T a\right) \varepsilon}{a^{2}}\left(\left(J^{2}+n\right)\left(J^{2} \sigma+n L s\right)-S_{C T} S_{T C} J^{4} L s \sigma\right)+R_{S} \sigma\left(\varepsilon S_{C T} J^{2}-\right.\right.$

$\left.\left.\left(J^{2}+n\right)\right)-\frac{R_{n} \sigma}{\left(J^{2} \sigma+n L n\right)}\left(\left(\left(J^{2}+n\right) L n+J^{2} N_{D} \varepsilon\right)\left(J^{2} \sigma+n L s\right)+S_{T C} J^{4} L s \sigma\left(L n S_{C T} \varepsilon+N_{D}\right)\right)\right]$

where $J^{2}=\pi^{2}+a^{2}$.

\section{The stationary convection}

The stationary convection will be characterized by $n=0$ in equation (31), and reduce it to

$R_{D}=\frac{1}{\left(\varepsilon-S_{T C} L s\right)}\left[\frac{J^{2}\left(-J^{2} P l+\pi^{2} T a\right) \varepsilon}{a^{2}}\left(1-S_{C T} S_{T C} L s\right)+R_{S}\left(\varepsilon S_{C T}-1\right)-R_{n}\left(\left(L n+N_{D} \varepsilon\right)+S_{T C} L S\left(L n S_{C T}+N_{D}\right)\right)\right]$

the thermal Darcy Rayleigh number reveal by equation (32) which is a function of $a, S_{C T}, S_{T C}, L e, N_{D}, R_{S}, R_{n}, L n$. Since elastico-viscous parameter $F$ vanish with $n$, so the Walters'(model B') elastico-viscous nanofluid react similar to usual Newtonian nanofluid, In the nonappearance of the Dufour and Soret parameters equation (32) reduces to

$R_{D}=\left[\frac{\left(\pi^{2}+a^{2}\right)\left(-\left(\pi^{2}+a^{2}\right) P l+\pi^{2} T a\right)}{a^{2}}-\frac{R_{S}}{\varepsilon}-R_{n}\left(\frac{L n}{\varepsilon}+N_{D}\right)\right]$

Here, take $x=\frac{a^{2}}{\pi^{2}}$, in equation (43), then we get 


$$
R_{D}=\pi^{2}\left[\frac{T a(1+x)}{x}-\frac{P l(1+x)^{2}}{x}\right]-\frac{R_{S}}{\varepsilon}-R_{n}\left(\frac{L n}{\varepsilon}+N_{D}\right)
$$

\section{Results and Discussion}

The equation (34) express for stationary thermal Rayleigh Darcy number compute as a function of solute Rayleigh number, nanoparticle Rayleigh number, modified diffusivity ratio, thermo-nanofluid Lewis number, Taylor number, medium permeability, porosity, and dimensionless wave number.

We observe the nature of $\frac{\partial R_{D}}{\partial T a}, \frac{\partial R_{D}}{\partial N_{D}}, \frac{\partial R_{D}}{\partial R_{n}}, \frac{\partial R_{D}}{\partial R_{S}}, \frac{\partial R_{D}}{\partial P l}$ and $\frac{\partial R_{D}}{\partial L n}$ analytically. Equation (34) gives $\frac{\partial R_{D}}{\partial T a}>0$ and $\frac{\partial R_{D}}{\partial R_{S}}<0, \frac{\partial R_{D}}{\partial N_{D}}<0, \frac{\partial R_{D}}{\partial P l}<0, \frac{\partial R_{D}}{\partial R_{n}}<0, \frac{\partial R_{D}}{\partial L n}<0$.

This implies that for stationary convection, Taylor number have stabilizing effect whenever Solute Rayleigh number, thermo-nanofluid Lewis number, modified diffusivity ratio, nanoparticle Rayleigh number and medium permeability have destabilizing effect on the system.

Figure 1 represents the Rayleigh Darcy number increase with Taylor number and for different values of solute Rayleigh number $R_{s}=100,200,300$ with the constant values of $N_{D}=1, P l=5, R_{n}=1, \operatorname{Ln}=1000, \varepsilon=$ 0.6. The Rayleigh number $R_{D}$ increase with the Taylor number $T a$, which implise that on the stationary convection Taylor number has stabilizing effect.

Figure 2 represents the Rayleigh Darcy number decrease with medium permeability and for different values of nanoparticle Lewis number $\operatorname{Ln}=1000,4000,7000$ with the constant values of $N_{D}=1, T a=100, R_{n}=$ $1, R_{S}=100, \varepsilon=0.6$. The Rayleigh number $R_{D}$ decrease with the medium permeability $P l$, which implise that on the stationary convection medium permeability has destabilizing effect.

Figure 3 represents the Rayleigh Darcy number decrease with solute Rayleigh number and for different values of Taylor number $\mathrm{Ta}=100,300,600$ with the constant values of $N_{D}=1, P l=5, R_{n}=1, \operatorname{Ln}=1000, \varepsilon=$ 0.6. The Rayleigh number $R_{D}$ decrease with the solute Rayleigh $R_{S}$, which implise that on the stationary convection solute Rayleigh has destabilizing effect.

Figure 4 represents the Rayleigh Darcy number decrease with nanoparticle Rayleigh number and for different values of diffusive ratio $N_{D}=1,5,10$ with the constant values of $T a=100, R_{S}=100, P l=5, \operatorname{Ln}=200, \varepsilon=$ 0.6. The Rayleigh number $R_{D}$ decrease with the nanoparticle Rayleigh number $R_{n}$, which implise that on the stationary convection nanoparticle Rayleigh number has destabilizing effect.

Figure 5 represents the Rayleigh Darcy number decrease with diffusive ratio and for different values of medium permeability $P l=1,5,10$ with the constant values of $T a=100, R_{s}=100, R_{n}=1, L n=200, \varepsilon=0.6$. The Rayleigh number $R_{D}$ decrease with the diffusive ratio $N_{D}$, which implise that on the stationary convection diffusive ratio has destabilizing effect.

Figure 6 represents the Rayleigh Darcy number decrease with nanoparticle Lewis number and for different values of nanoparticle Rayleigh number $R_{n}=1,2,3$ with the constant values of $T a=100, R_{s}=100, P l=$ $1, N_{D}=1, \varepsilon=0.6$. The Rayleigh number $R_{D}$ decrease with the nanoparticle Lewis number $R_{n}$, which implise that on the stationary convection nanoparticle Lewis number has destabilizing effect. 


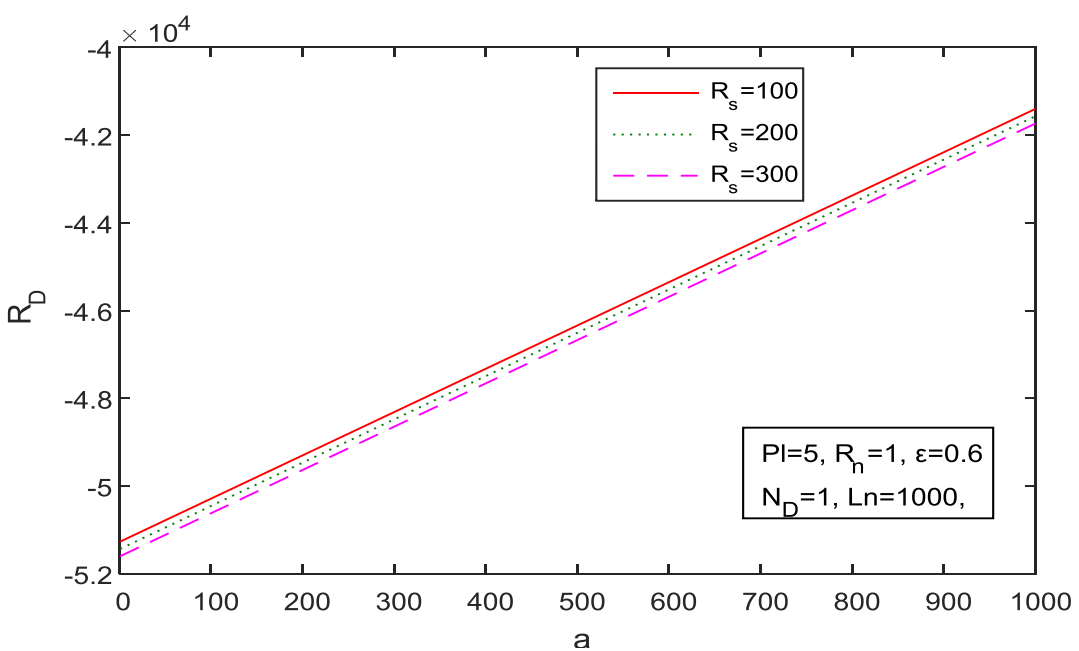

Figure: 1

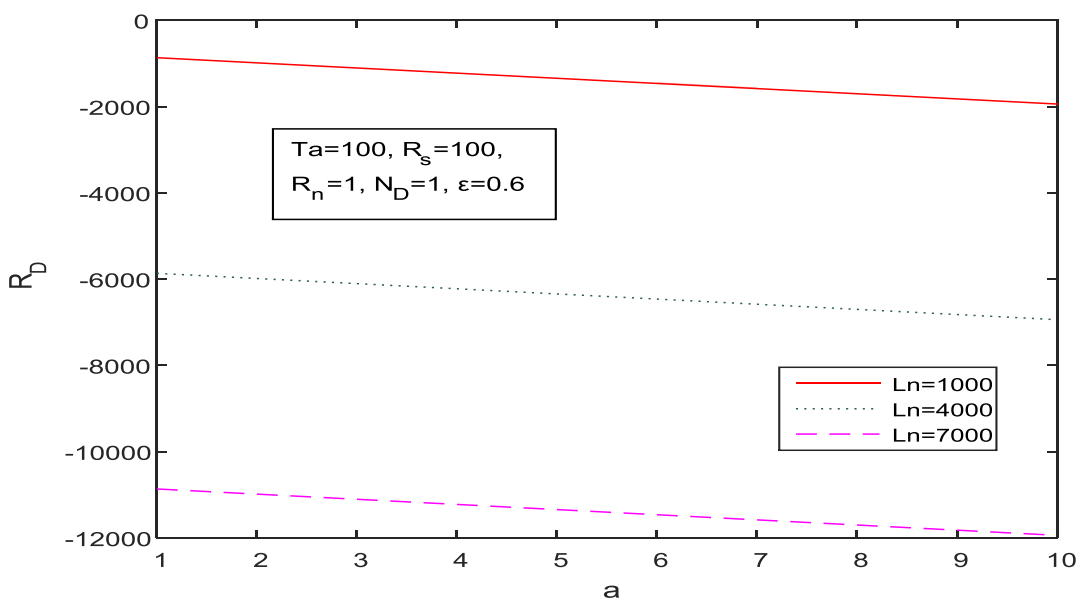

Figure: 2

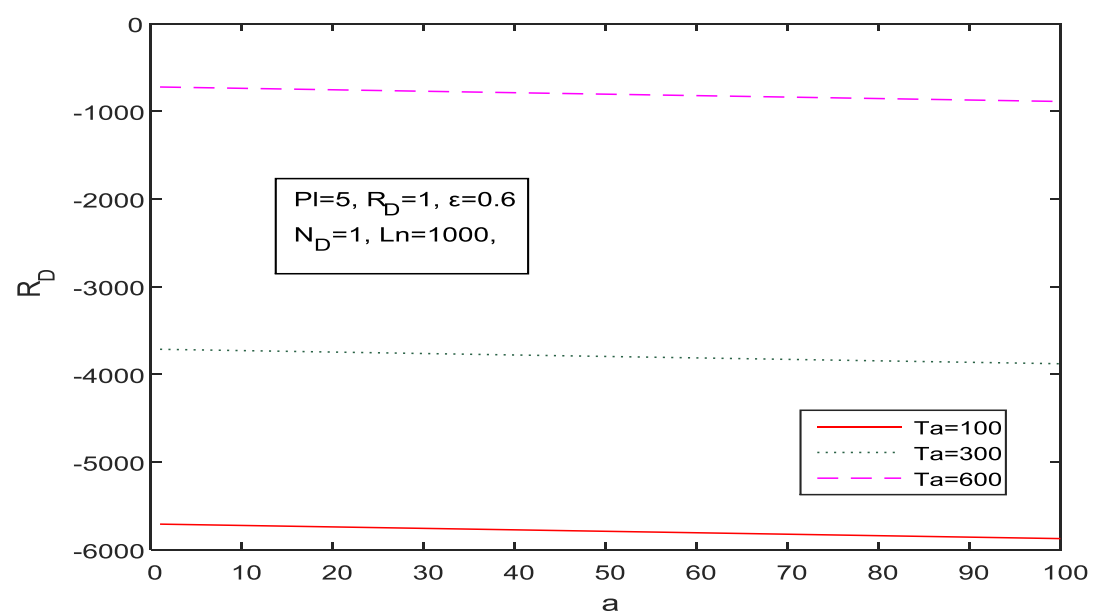

Figure: 3 


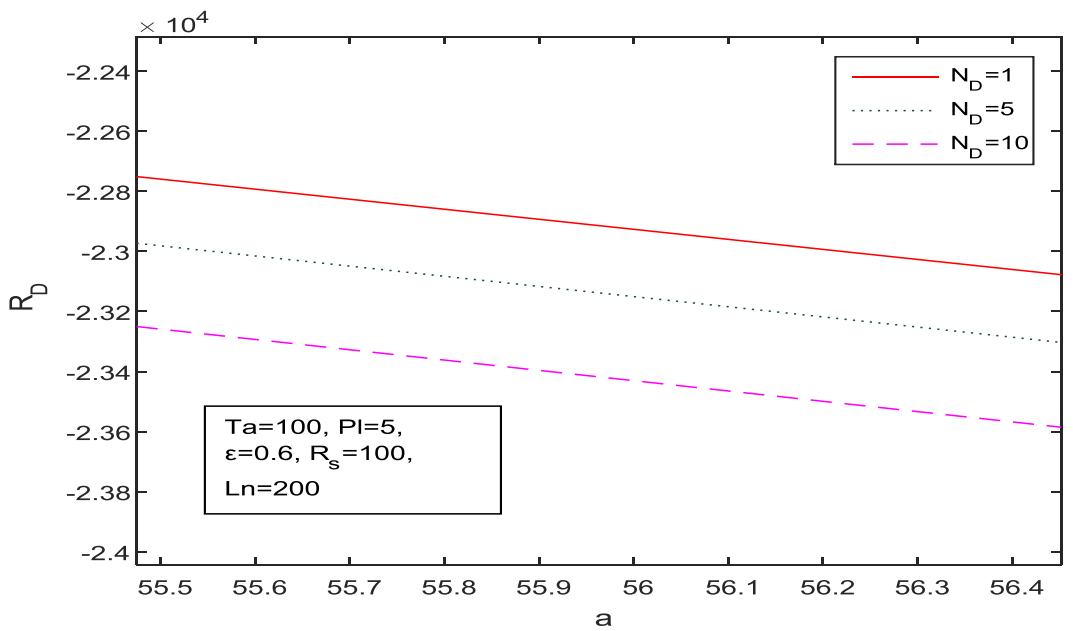

Figure: 4

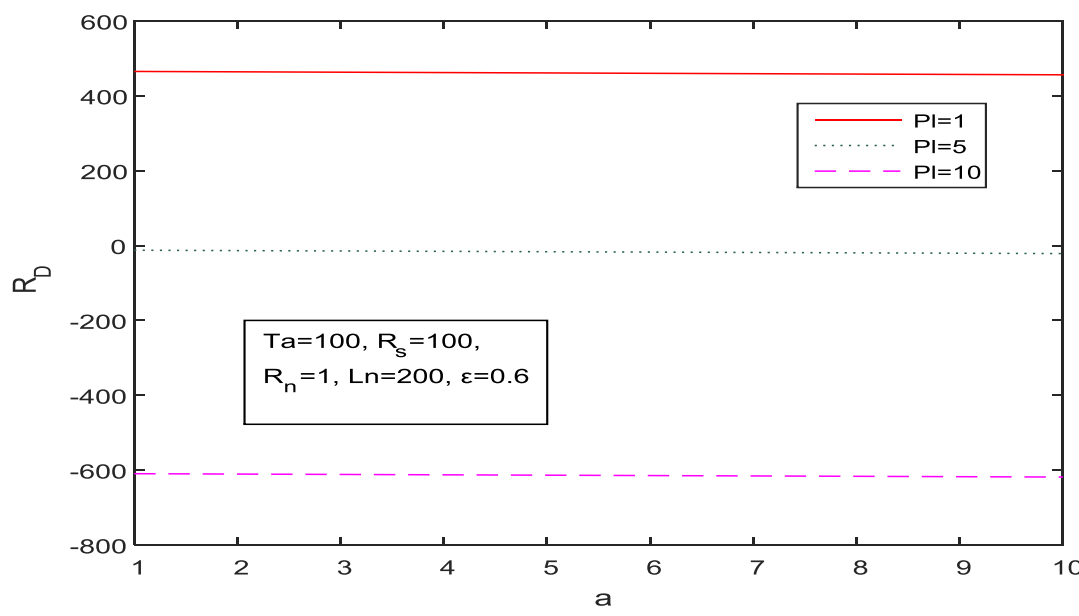

Figure: 5

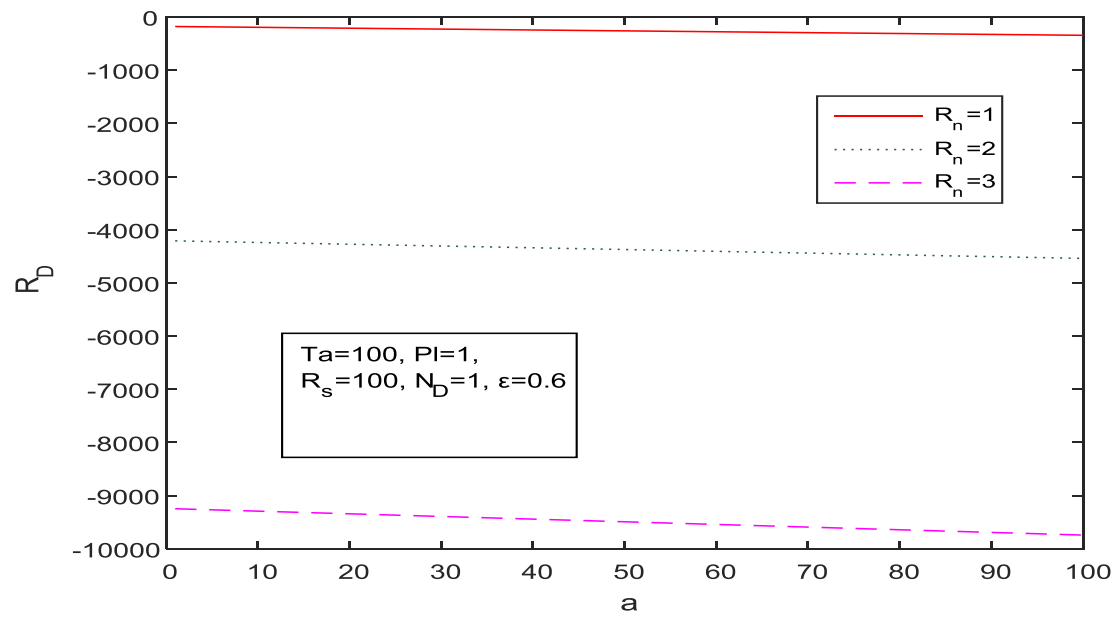

Figure: 6

\section{Conclusion}

The effect of rotation on thermosolutal convection of visco-elastic nanofluid with porous medium using Walters` (Model B`) is investigated by using linear stability analysis. We drawn the main conclusion are following as: 
(i) Due to rotation, Taylor number has stabilizing effect for stationary convection.

(ii) Solute Rayleigh number, thermo-nanofluid Lewis number, modified diffusivity ratio, nanoparticle Rayleigh number and medium permeability have destabilizing effect for stationary convection.

The Walters` (model B`) elastico-viscous nanofluid react similar to regular Newtonian nanofluid for stationary convection.

\section{References}

(1) Buongiorno, J., "Convective transport in nanofluids, " ASME Journal of Heat Transfer, vol. 128 (3), pp 240-250, 2006.

(2) Chandrasekhar S., "Hydrodynamic and hydromagnetic stability," Dover Publication, New York, 1981.

(3) Chand R. and Rana G. C., "On the onset of thermal convection in rotating nanofluid layer saturating a Darcy-Brinkman porous medium, "International Journal of Heat and Mass Transfer, vol. 55, pp 54175424, 2012.

(4) Choi S., "Enhancing thermal conductivity of fluids with nanoparticles," In D.A. Siginer, H.P. Wang (Eds.), Development and Applications of Non-Newtonian Flows, ASME FED- 231/MD vol. 66, pp 99105, 1995.

(5) Gupta U., Sharma J. and Wanchoo R. K., "Thermosolutal convection in a horizontal nanofluid layer: Introduction of oscillatory motion, Recent Advance in Engineering and Computation Science, IEEE, Chandigarh, India, Print ISBN 978-1-4799-2290-1, 2014.

(6) Kuznetsov A.V. and Nield A. V., "the onset of double diffusive nanofluid convection in a layer of a saturated porous medium," Transp. Porous Medium, vol. 85, pp 941-951, 2010.

(7) Nield A. V. and Kuznetsov A.V., "the onset of double diffusive convection in a nanofluid saturated porous medium," International journal of Heat and Fluid Flow, vol 34, pp 771-776, 2011.

(8) Pundir S. K., Kumar M. and Pundir R., “ on the onset of thermosolutal convection of an elastic-viscous nanofluid in porous medium in presence of magnetic field, "Compliance Engineering Journal, vol. 11, pp 104-112, 2020

(9) Rana G.C. and Sharma V., "Hydromagnetic thermosolutal instability of Walters' (model B') rotating fluid permeated with suspended particles in porous medium," The International Journal of Multiphysics, vol. 5, pp. 325-338, 2011.

(10) Sharma J. and Gupta U, "Double diffusive nanofluid convection in porous medium with rotation: Darcy-Brinkman model," Procedia Engineering, vol. 127, pp 783-790, 2015.

(11) Yadav D., Agarwal G. S, and Bhargav R., "the onset of convection in a binary nanofluid saturated layer," International Journal of Theortical and Applied Multiscale Mechanics, vol. 2, pp 198-224, 2012. 